\title{
Vibration Signal Analysis of High Voltage Circuit Breakers System Based on Hybrid Model of Power Spectrum Estimation
}

\author{
Fu Chao ${ }^{1,2, *}$ \\ ${ }^{1}$ Hebei Normal University, Shi Jiazhuang, 050024, China \\ ${ }^{2}$ Province-Ministry Joint Key Laboratory of Electromagnetic Field and Electrical Apparatus Reliability, Hebei Univer- \\ sity of Technology, Tianjin, 300130, China
}

\begin{abstract}
This paper discusses several methods of power spectrum estimation, respectively using the indirect method and Welch method to estimate power spectrum distribution through the acquisition of vibration signals of circuit breakers, and use the AR hybrid model to estimate different orders of power spectrum estimation. Under different length of data, the order selection method can be determined.
\end{abstract}

Keywords: Spectrum estimation, vibration signal, AR model.

\section{INTRODUCTION}

Power spectrum estimation method is a power spectrum calculation method of vibration or random signal. It is an important link in the signal analysis, which can be used to detect the periodic component and the percentage of the signal [1]. And it is the great significance for the diagnosis of mechanical failure. Using a given set of sample data to estimate the power spectral density of a stationary random signal is called power spectrum estimation. Power spectral density is the discrete time Fourier transform of the random signal autocorrelation sequence, it is used to characterize the statistical properties of random signals. The power spectrum of random signal reflects its frequency components and the relative strength of each component, it can reveal the signal rhythm from the frequency domain, and it is the important characteristic of random signals.

Found in the study, statistical properties of $\mathrm{CB}$ vibration signal under different working conditions, the results show that when the circuit breaker is in normal working condition or state change is very small, the action of mechanical vibration signal can be approximated at a stationary random process, which is consistent with the $A R$ hybrid model assumption is usually described by. Therefore, the $A R$ hybrid model power spectrum estimation can achieve the desired effect of vibration signal.

Generally speaking, the power spectrum estimation method can be divided into classical spectral estimation method and modern spectral estimation method. Classical spectral estimation method is defined directly by using the finite-length sequence to estimate, there are two ways to achieve:
(1) Estimate the correlation function first, and then get the power spectrum estimation by the Fourier transform.

(2) Link the power spectrum and the square of the signal amplitude-frequency characteristics [2]. No matter which way to use, the common problem is that the estimation of variance characteristic is not good, and the estimated value of ups and downs along the frequency axis is very fierce. This phenomenon is more serious as the longer of the data.

Classical spectral estimation method can be divided into direct and indirect methods, direct method is to use a fast Fourier algorithm (FFT) for a finite number of sample data to obtain a power spectrum of the Fourier transform method, and it is also called periodogram; The indirect method is getting the autocorrelation function estimation of sample data in the first, then performing Fourier transform to obtain the power spectrum. Since direct method to obtain the power spectrum estimation presences some shortcomings such as spectral curves undulating is volatile, spectral resolution is not high, several improved algorithms are proposed, such as Bartlett method and Welch method. Modern spectral estimation is mainly aimed at the low resolution spectral estimation variance and poor performance and other issues of the classical spectral estimation method, from the way the modern spectral estimation method can be divided into parametric model method and non-parametric model method, power spectrum estimation based on parametric modeling is an important content of modern power spectrum estimation. Here are several main parameters of the model method: $A R$ hybrid model method, $M A$ and $A R M A$ hybrid model method model law [3].

\section{CLASIC SPECTRUM ESTIMATION}

\subsection{Direct Method's Improved Algorithms}

For the direct method to obtain the power spectrum estimation, when the data length is too large, the spectrum 
curves undulating; if the data length is too small, the spectral resolution is not high [4], So people also proposed several improved algorithms, such as Bartlett method and Welch method.

\section{1) Bartlett method}

Bartlett average periodogram method is getting $N$ points finite-length sequence $x(n)$ segments seeking re average. Assuming that $x(n)$ into $L$ segments, and $M$ samples per segment, Thus $N=L M$, paragraph i sample sequence can be written as:

$x^{i}(n)=x(n+(i \quad 1) M) 0 \leqslant n \leqslant M \quad 1,1 \leqslant i \leqslant L$

$\hat{p}_{p e r}^{i}(\omega)=\frac{1}{M}\left|\sum_{n=0}^{M} x^{i}(n) e^{j \omega n}\right|^{2}$

2) Welch method

Welch method of Bartlett law was amended in two respects: First, select the appropriate window function $\omega(n)$, and directly adding in front of the cycle diagram calculation, so that the resulting cycle Pictured each segment:

$\hat{p}_{p e r}^{i}(\omega)=\frac{1}{M U}\left|\sum_{n=0}^{M} x_{n}{ }^{i}(n) \omega(n) e^{j \omega n}\right|^{2}$

in the formula,

$U=\frac{1}{M} \sum_{n=0}^{M} \omega^{2}$

Is normalization factor. Windowed advantage is that no matter what kind of window functions can make nonnegative spectrum estimation; and, when segmented, can overlap between segments, this would reduce the variance.

Corresponding to the sum, and then averaged to obtain an average is the power spectrum:

$\bar{P}_{\text {pre }}=\frac{1}{L} \sum_{i=1}^{L} p_{\text {per }}^{i}(\omega)$

\subsection{The Indirect Method}

The indirect method estimates the correlation function by the sequence $x(n)$ first, Then do Fourier transform of the autocorrelation function evaluation [5], we get the power spectrum estimation of sequence $x(n)$, namely:

$\hat{p}_{B T}(\omega)=\sum_{m=N}^{N} \hat{R}_{x}(m) e^{j \omega n}$

\subsection{Simulation Analysis}

Take a random sequence of noisy $x(n)=4 \mathrm{cos}$ $100 \pi n+7 \cos 200 \pi n+z(n), z(n)$ is a random white noise, Several methods were used at the power spectrum estimation, as well as to facilitate comparison, the power spectral density do normalization, as shown in Fig. (1).
Although the Bartlett method does not specify window function, but for finite-length sequence, the equivalent rectangular windows; meanwhile to facilitate comparison, Welch method also takes a rectangular window.

From the spectral estimation effect, the resolution of the indirect method is significantly better than the direct method; Comparison of Fig. (1e, f), we can see that Welch method's spectral estimation's error is better than Bartlet's [6]. This is because although have adopted the rectangular window, But in the algorithm, when segments the same sequence, Welch method have overlap between the paragraphs, but Bartlett method didn't. The experiments show that Overlapping $1 / 2$ data length is appropriate, therefore, the estimated variance characteristics, the former than the latter; If the window function better replaced Hamming window, Blackman window, using the Welch method was relatively smoother curve, as shown in Fig. (1)

Take a group of scene acquisiting breaker vibration signal, using the indirect method, Welch method to estimate the power spectrum distribution respectively Fig. (2).

Despite the length of the vibration signal reaches $N=1500$, can be counted as long data samples, Spectrum estimation results are still not satisfactory. Analysis found that although Welch method does a considerable improvement, but still essentially unknown outside the work area data assume that the data is zero, equivalent data windowing, resulting in reduced resolution and spectral estimation instability; The indirect method is the autocorrelation function of the sequence directly as Fourier transform, so the impact will be better than the Welch method [7].

Modern spectral estimation methods have no longer simply assumed the unknown data observed outside the region and to be zero, but first estimate the model parameters through observation data signals, and estimate signal power spectrum in accordance with the output power of a model, which avoids the data hypothetical question outside the data observation area, and the spectrum estimation performance is superior to the classical spectrum estimation. Therefore, the following attempts to adopt modern spectral estimation methods Fig. (3).

\section{AR HYBRID MODEL OF THE POWER SPEC- TRUM ESTIMATION}

The power spectrum estimation based on the parametric modeling is an important part of modern power spectrum estimation, which the purpose is to improve the frequency resolution of the power spectrum estimation. Modern spectral estimation is parametric model spectrum estimation method based on random process. It can also be called a parametric model approach or simply model approach. Spectrum estimation method based on the general parameters of the model according to the following three steps:

(1) To be determined or estimated random process to select a reasonable model; 


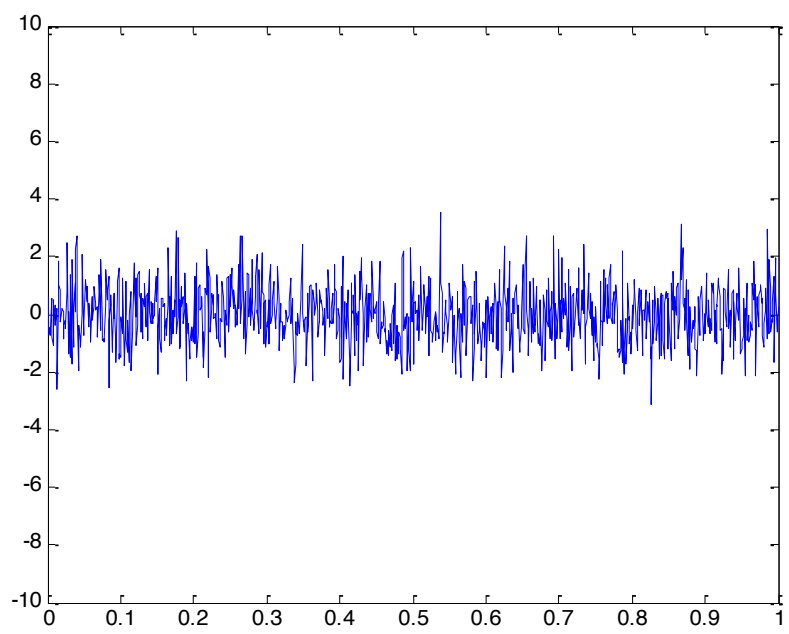

(a) Time Domain

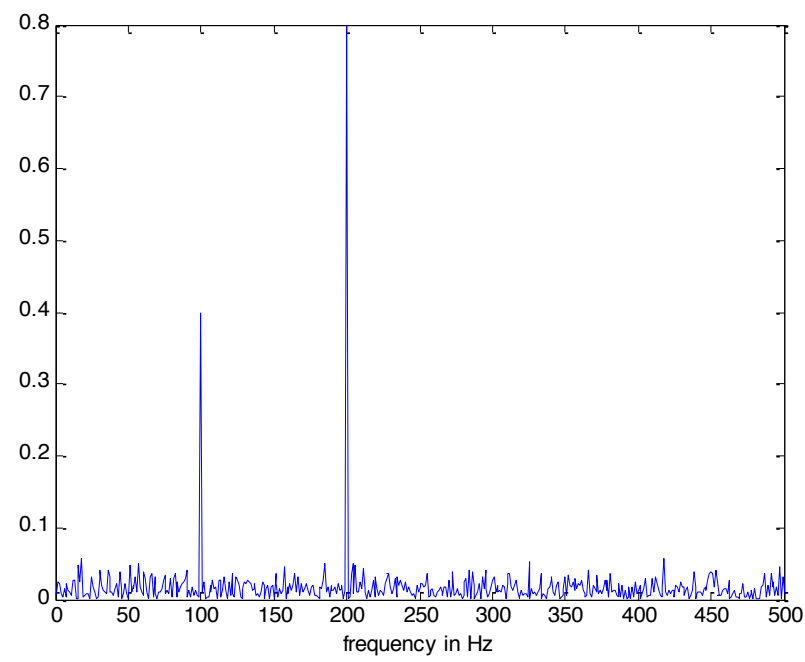

(b) FFT Spectrum Analysis

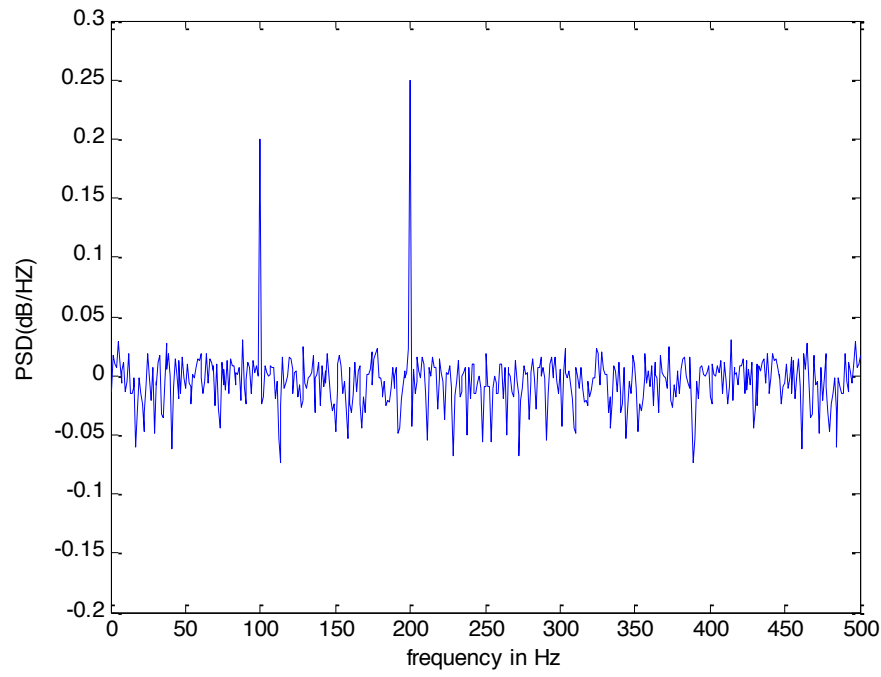

(c) Direct method 


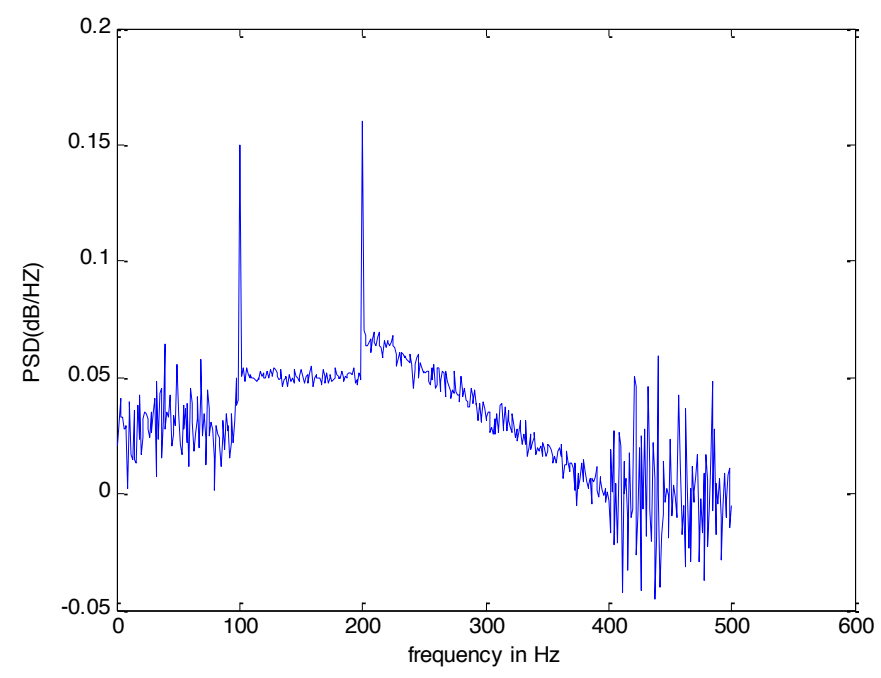

(d) Indirect method

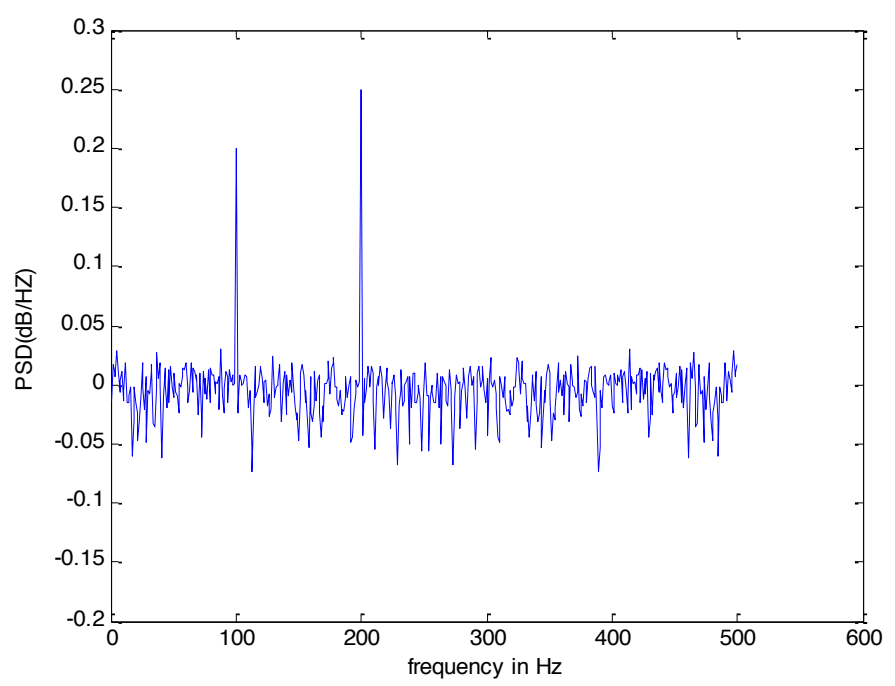

(e) Bartlett method

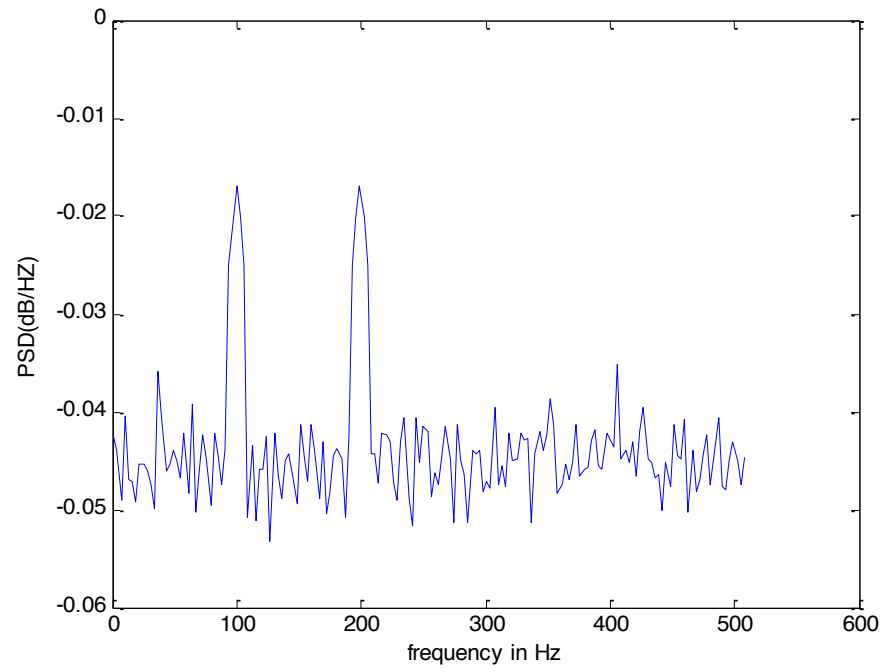

(f) Welch method

Fig. (1). Comparative sequence $\mathrm{x}(\mathrm{n})$ of different power spectrum estimation. 


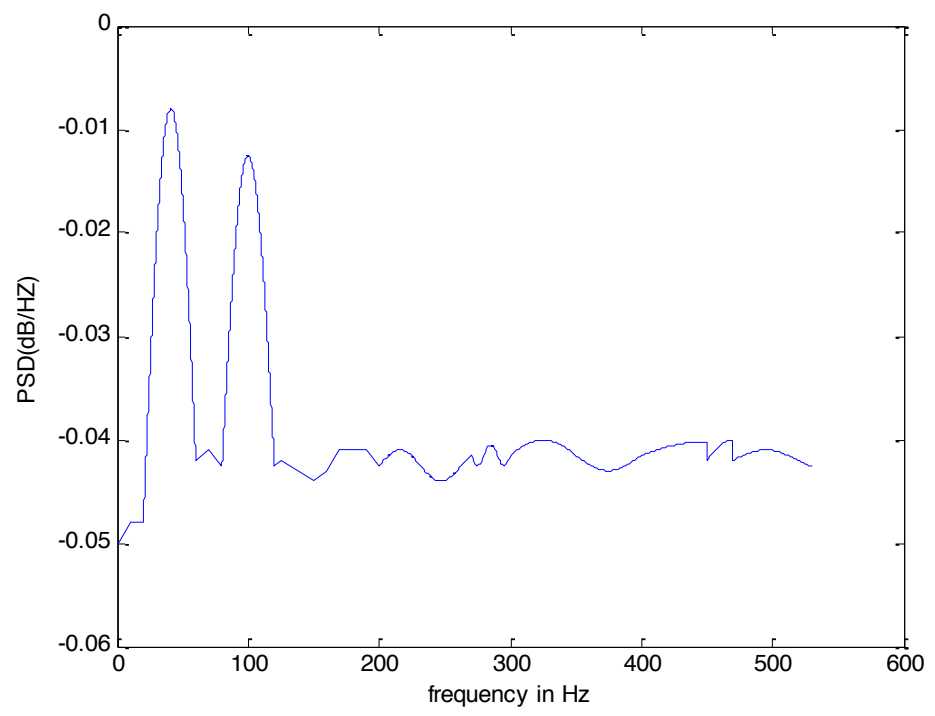

(a) Hamming window

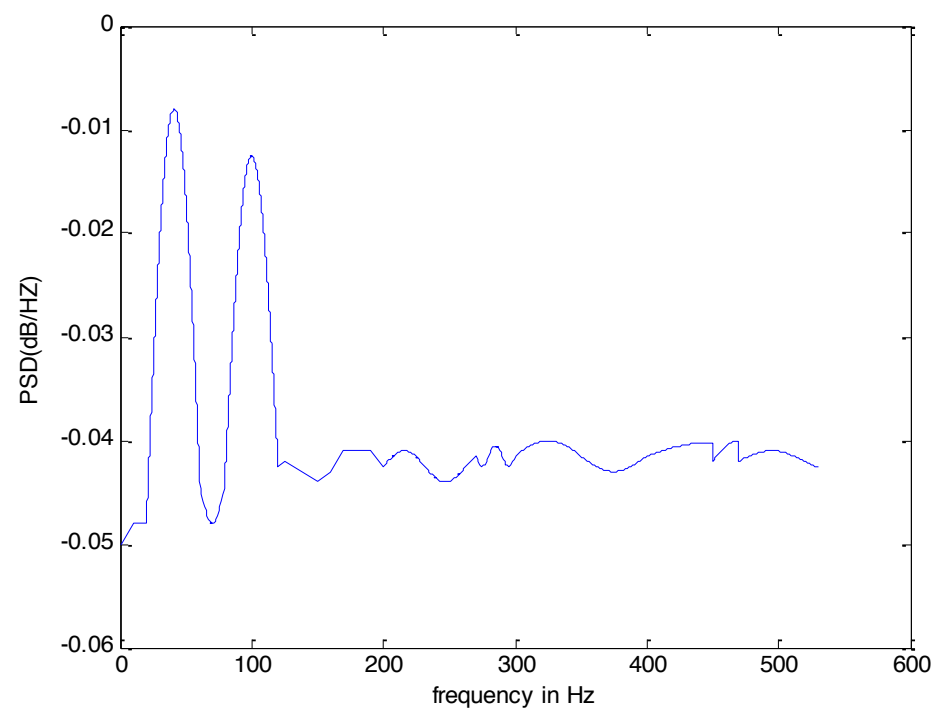

(b) Gabe Blackman window

Fig. (2). Welch power spectrum estimation method.

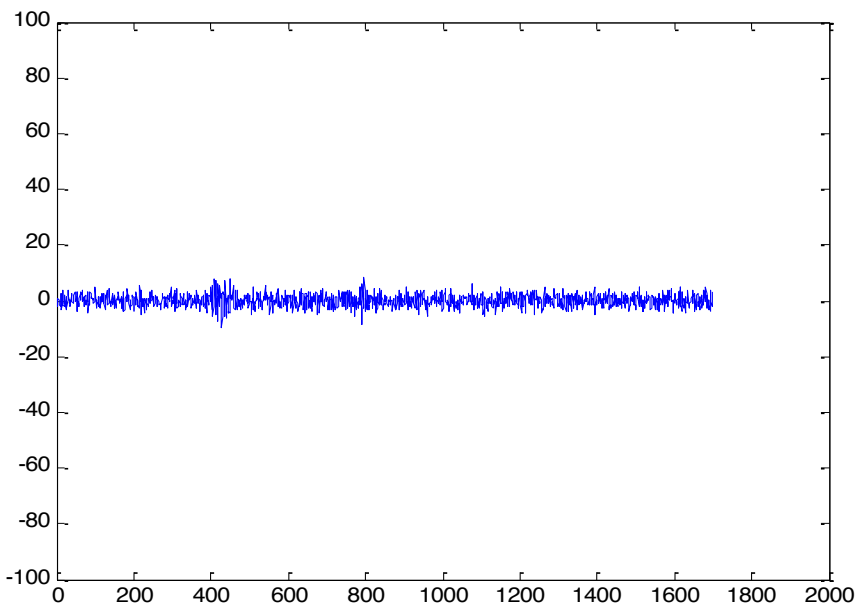

(a) The original signa 


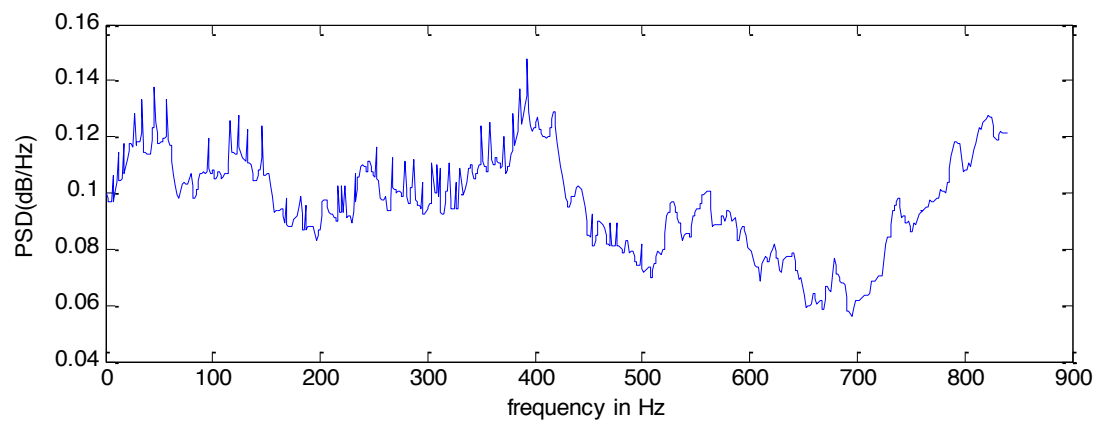

(b) FFT spectrum analysis

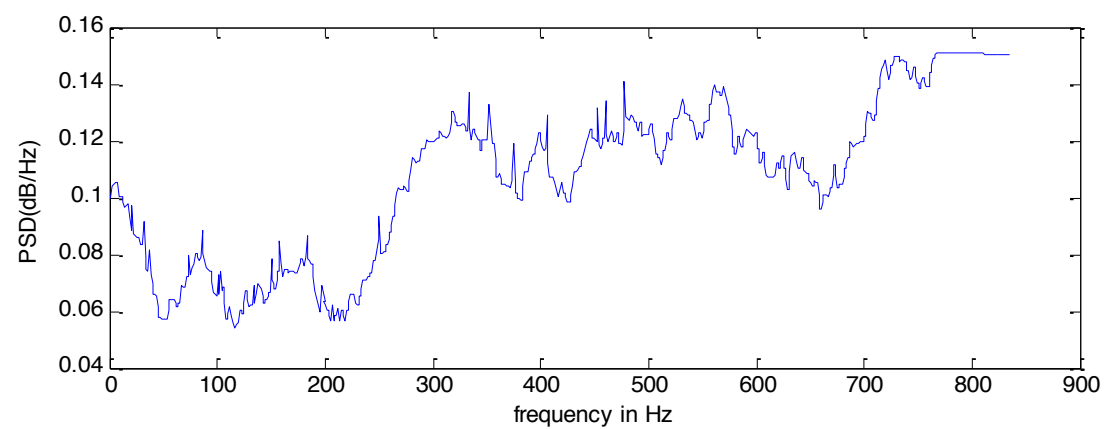

(c) The indirect method

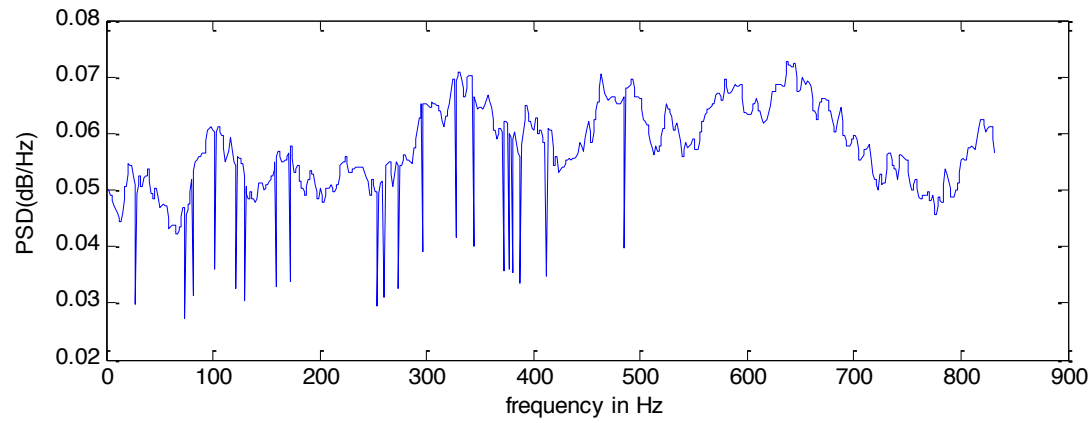

(d) Welch Act (Gabe Blackman window)

Fig. (3). Two power breaker vibration signal spectrum estimation.

(2) Model parameter estimation based on known observation data;

(3) Model parameter estimation was used to calculate the power spectrum [8].

Modern spectral estimation model is divided into $A R$ hybrid model, $M A$ hybrid model and the $A R M A$ hybrid model. $M A$ hybrid model, also called moving average model, is the all-zero hybrid model; $A R M A$ hybrid model is also known as auto-regressive moving average model, the "polezero" models. ARMA or $M A$ course of any process can be represented by an infinite order. Even if you choose an appropriate model spectrum estimation procedure, you can always possible to select a sufficiently high order of approximation to the stochastic process modeled [9]. Since accurate estimation of $A R$ hybrid model parameters can be obtained by solving a set of linear equations, and for $M A$ and $A R M A$ hybrid model of the power spectrum estimation, accurate estimation of its parameters need to solve a set of higherorder nonlinear equations. Practice shows, $A R$ hybrid model has a power spectrum for short data analysis, calculation is simple, get a smooth spectral resolution advantages, therefore $A R$ hybrid model has been widely used.

\subsection{AR Hybrid Model of the Power Spectrum Estimation Fundamentals}

A number of stationary random processes can be generated by using white noise excitation linear time-invariant systems. Fig. (4), $x(n)$ is composed of an input sequence 


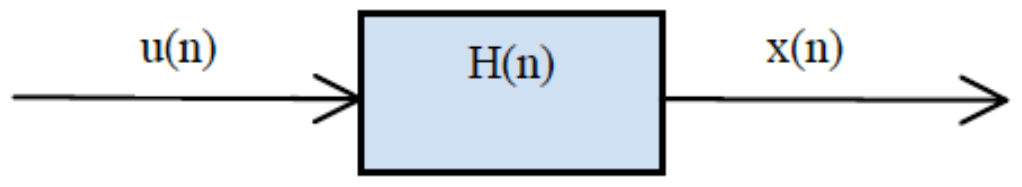

Fig. (4). Parameters of the system model.

$u(n)$ excitation output of a causal linear shift invariant discrete-time system $H(n)$.

If the discrete stationary random sequence $x(n)$ can be linear differential equation (8) to describe:

$x(n)+\sum_{i=1}^{p} a_{i} x(n \quad i)=u(n)+\sum_{k=0}^{q} b_{k} e(n k)$

Where $u(n)$ is a discrete white noise, called $\{x(n)\}$ is ARMA process.

Formula (7) on both sides is taken $\mathrm{Z}$ transform, and assuming $b_{0}=1$, can be obtained by formula (8), that is

$H(z)=\frac{B(z)}{A(z)}=\frac{\sum_{k=0}^{q} b_{k} z^{k}}{\sum_{k=0}^{q} a_{k} z^{k}}$

of formula (8)

$A(z)=1+\sum_{k=1}^{p} a^{k} z^{k}$

$B(z)=1+\sum_{k=1}^{q} b^{k} z^{k}$

Thereby obtaining the output sequence $x(n)$ is a power spectrum

$p_{x}\left(e^{\star j \omega}\right)=\frac{\sigma^{2}\left|B\left(e^{\star i \omega}\right)\right|^{2}}{\left|A\left(e^{\star j \omega}\right)\right|^{2}}$

If $b_{1}, b_{2}, \ldots, b_{q}$ are all zero, the formula (8) becomes:

$x(n)=\sum_{k=1}^{p} a_{k} x(n k)+u(n)$

This model is called autoregressive model, referred to as $A R$ hybrid model. Where $u(n)$ is a white noise with variance $\sigma^{2}$ sequence, $\mathrm{p}$ is the order of the $A R$ hybrid model, $a_{k}$ the $A R$ hybrid model parameters, $K=1,2, \ldots p$. For the $A R$ hybrid model, signal $x(n)$ from the previous value itself several excitation current value and generating a linear combination, and the accurate estimation of $A R$ model parameters can be obtained by solving a set of linear equations [10].

Also, the formula (11) becomes:

$$
p_{X}\left(e^{\star j \omega}\right) \frac{\sigma^{2}}{\left|1+!_{k=1}^{p} A a_{k} e^{j \omega k}\right|^{2}}
$$

That is the random signal $x(n)$ of $A R$ hybrid model of the power spectrum. It can be seen, to power spectrum estimation, we must obtain the $A R$ hybrid model order $p, A R$ hybrid model parameters $a_{1}, a_{2}, \ldots a_{p}$ and $\sigma^{2}$.

\subsection{Choose the AR Hybrid Model Order}

Select the appropriate $A R$ hybrid model order AR spectral estimation is a key issue, Order $p A R$ hybrid model is generally not known in advance, you need a slightly larger pre-selected value, determined in the recursive process. When using the Levinson recursive algorithm (LevinsonDurbin), can be given by the low-level to high-end of each set of parameters, and the minimum prediction error model of the power $p_{\min }$ (equivalent to white noise sequence variance $\sigma^{2}$ is decreasing. Intuitively, $p$ when the prediction error reaches a specified desired value, or no longer change, then the correct order of the order that is to be selected.

Because prediction error power $p$ is a monotonically decreasing, this value is reduced to just the right number is often hard to choices, Order selection too low will have a greater bias, and may cause peaks can't tell; Select the parameters to be estimated too high is because too much performance degradation caused by spectral estimation variance. To this end, there are several different criteria have been proposed, the following are a few of the more common criteria.

1) Several common criteria to determine the AR hybrid model order

a) Final prediction error criterion ( $F P E$ )

Make $\operatorname{FPE}(r)$ for the application of the minimum value of the order of $r$

$F P E(r)=P_{r} \frac{N+r+1}{N r 1}$

Where $\mathrm{r}$ is the order of the prediction error $P_{r}$ power, $N$ is the number of samples, according to the following formula to calculate $P_{r}[11]$.

$p_{r}=\sum_{j=0}^{r} a_{j} R(j)$ 
Table 1. Random distribution of each criterion with random Gaussian white noise signal number order.

\begin{tabular}{|c|c|c|c|c|}
\hline $\mathbf{i}=\mathbf{1 , 2 , 3 , 4}$ & AIC Criterion & FPE Criterion & MDL Criterion & Empirical formula \\
\hline \hline $\mathrm{Pi}$ & 42 & 42 & 26 & 26 \\
\hline $\mathrm{Pi}$ & 41 & 41 & 21 & 26 \\
\hline $\mathrm{Pi}$ & 50 & 50 & 20 & 26 \\
\hline $\mathrm{Pi}$ & 42 & 42 & 26 \\
\hline
\end{tabular}

$a_{j}$ as a model parameter, $R(j)$ of the signal $x(n)$ of the autocorrelation function.

b) Information criterion ( $A I C$ )

Make $A I C(r)$ for the application of the minimum value of the order of $r$.

$$
A I T(r)=N \ln \left(p_{r}\right)+2 r
$$

Wherein the parameters $N, P_{r}$ are same as defined above. When the order $\mathrm{r}$ of a start to increase, $\operatorname{FPE}(r)$ and the $A I C(r)$ are obtained at a certain minimum value of the $r$, $\mathrm{r}$ at this time is the most appropriate as the order $\mathrm{p}$.

When the data is shorter, FPE, AIC given the low number of bands, and the results are given in the two is basically the same. When the sample size $N$ tends to infinity, the amount of information $F P E(r)$ and $A I C(r)$ equivalent, when $N \rightarrow \infty$, AIC criteria for selecting the correct order of the probability of error is not zero [12].

c) The minimum description length $(M D L)$

Rissanen proposed an alternative information criterion minimum description length ( $M D L), M D L$ criterion is based on the process to make the model corresponding probability distribution probability approaching the maximum possible distribution of the actual process of thinking. It is defined as the amount of information.

$\operatorname{MDL}(r)=N \ln \left(P_{r}\right)+r \ln N$

$M D L$ statistical information criterion is consistent, experimental results show that the short data, $A R$ order should be selected in the $N / 3 \sim N / 2$ range will have good results.

Clayton Ulrych and proved that for a short segment, FPE, AIC work was not good.

d) The empirical formula

Engineering frequently using the following formula:

$P=\frac{2^{1 * k} N}{\ln \left(2^{1 * k} N\right)}$

Where $N$ is the length of the data signal, $k$ is the wavelet packet decomposition level.
According to the formula shows that order of a), b), c) three criteria estimates on the prediction error power $P_{r}$, by the formula (15), $P_{r}$ consists of a sequence $x(n)$ of the autocorrelation function $r(j), A R$ hybrid model parameters $a(j)$ two parts; According to the definition of both, when the sequence $x(n)$ changes in the autocorrelation function $r(j), A R$ hybrid model parameters $a(j)$ values also changed [13]; The empirical formula is defined by commonly known, order $p$ by the sequence length $N$, the number of wavelet packet $k$ decisions and sequence values, the noise itself is irrelevant; so the four methods, in addition to commonly used empirical formula, the order $p$ under the other three criteria were randomly change (see Table 1), Wherein i is a random number of operations, $\mathrm{i}=4$.

It should be noted that the above is only a few criteria for order selection provides a basis, for the study to a specific signal, and ultimately choose their $A R$ hybrid model order, you need to practice to compare the results of multiple [14].

\section{SIMULATION ANALYSIS}

Take a sequence of noisy $x(n)=3 \cos (200 \pi n)$ $+5 \cos (100 \pi n)+e(n), \quad e(n)$ is a random Gaussian white noise, sampling rate $f_{s}=100 H Z$, take $N=1024$ points, four methods are solved on $A R$ hybrid model parameters $p$, the results in Table $\mathbf{1}$.

As can be seen by the sequence $x(n)$ of the expression, because $e(n)$ is a random Gaussian white noise, random values generated by each operation, so that the sequence $x(n)$ values have changed.

Take another noisy sequence $x(n)=3 \sin (400 \pi n)$ $+5 \cos (402 \pi n)+x_{e}(n), x_{e}(n)$ is a noisy square wave, $x_{e}(n)$ is not randomly distributed, In the same bandwidth (assuming here are $[0,1])$ of the premise, change the sample rate $f_{s}$ (equivalent to changing the sequence length $N$ ), the results in Table 2.

Found from Table 2, the sequence $x(n)$ by a longer data ( $N=2048)$ becomes short data $(N=32)$ of the process, the 
Table 2. The following four kinds of sequences of different lengths in order to determine.

\begin{tabular}{|c|c|c|c|c|}
\hline $\mathbf{N}$ & AIC Criterion & FPE Criterion & MDL Criterion & Empirical formula \\
\hline \hline $\mathrm{P}(\mathrm{N}=2048)$ & 77 & 77 & 11 & 26 \\
\hline $\mathrm{P}(\mathrm{N}=1024)$ & 50 & 50 & 9 & 18 \\
\hline $\mathrm{P}(\mathrm{N}=512)$ & 28 & 28 & 7 & 9 \\
\hline $\mathrm{P}(\mathrm{N}=256)$ & 17 & 17 & 8 & 6 \\
\hline $\mathrm{P}(\mathrm{N}=128)$ & 8 & 8 & 1 & 4 \\
\hline $\mathrm{P}(\mathrm{N}=64)$ & 8 & 5 & 1 & 3 \\
\hline $\mathrm{P}(\mathrm{N}=32)$ & 5 & 8 & \\
\hline
\end{tabular}

method of determining the order of four substantially decreased. Detailed analysis of the following variation of the order under $A I C, F P E, M D L$ criterion $p$ 's [15]:

(1) $A I C$ criteria and guidelines for the $F P E$, regardless of size $N$ samples, the results given both are the same, when the number of samples $N$ is shorter, the order declined rapidly, but compared with the other two methods, the value is relatively high;

(2) For $M D L$ criterion when short data is much smaller than the estimated value given by $N / 3 \sim N / 2$ range, the power spectrum estimation done, the frequency resolution is very poor, or even not affect the resolution of frequency components; For short data, $A R$ order should be selected in the $N / 3 \sim N / 2$ range will have good results. I try several randomly noisy sequence analysis results of many experiments show that when changes in the order $N / 3 \sim N / 2$ range, the result is the estimation of each $[\mathrm{N} / 3]$.

Take the following sequence of noisy $x(n)=3 \cos \left(2 \pi \times f_{1} \times n\right)+5 \cos \left(2 \pi \times f_{2} \times n\right)+0.1 e(n) \quad$, $f_{1}=8 H Z, f_{2}=12 H Z, e(n)$ is a Gaussian white noise, four methods ( $A I C, F P E, M D L$, commonly used empirical formula) results are given as follows: $P_{1}=P_{2}=6 ; P_{3}=5$; ; $P_{4}=3$; Here Conclusion Based on the above (2) said, adding a case under that $M D L$ criteria when short data, order $P_{3}^{\prime}$ take $[N / 3]$; In determining the order of the $A R$ hybrid model $r$ premise Burg method using the estimated power spectrum, spectral estimation results shown in Fig. (5).

When the data sequence is short, that is $N=32$, the order of $A I C, F P E$ standards and guidelines for obtaining similar $M D L[16]$, when the order of $P_{1}, P_{2}, P_{3}$ were taken Fig. $(\mathbf{1 b}, \mathbf{c})$ the power spectrum of the frequency curve can be clearly distinguished $f_{1}=8 H Z, f_{2}=12 H Z$ two peaks
[17], this is consistent with the results of (a) the fast Fourier algorithm (FFT) spectrum analysis; the results of empirical formula used $P_{4}=3$, the spectral curve is too smooth [18], the spectral resolution is poor, unable to distinguish $f_{1}=8 H Z, f_{2}=12 H Z$ two peaks Fig. (5e), this is because we can see by the empirical formula, the results of the order $P_{4}$ only by the signal length $N^{[19]}$, wavelet packet decomposition level $\mathrm{k}$ to decide, regardless of the frequency components of the signal; Fig. (5d) $M D L$ criterion is also used, the order taking $P_{3}^{\prime}[\mathrm{N} / 3]=10$, the effect of the power spectrum estimate (b), (c), as can clearly distinguish the two frequency peaks; Note that, in order to continue to increase during $P_{3}^{\prime} \mathrm{N} / 2$, the spectrum smoothing section undulating curve intensified, new peaks appeared, the spectrum changes shown in Fig. (6).

When the order $\mathrm{r}$ to 13 , starting at $f \approx 4 H Z$ spectrum spurious peaks (see Fig. (6e)); continue to increase $r$, the spectrum has been found in more false peaks, Fig. (6d-e) shown below, but the pseudo-peak energy relative to the two main peaks appear ( $f_{1}=8 H Z, f_{2}=12 H Z$ Department) is very weak, from the curve was still able to clearly distinguish the two peaks [17-23].

Therefore, when a shorter data using $M D L$ criterion, the order can be directly taken $[N / 3$, also available at $(N / 3$, within the range of $N / 2$ ) values, spectral estimation results are basically unaffected.

\section{CONCLUSION}

Based on the above analysis, although some guidelines have been proposed, but no single criterion can be well suited to a variety of situations, several selection criteria described in the order of only provide a basis for determining the order of the final signal is often affected by the specific nature of the study, the analysis of the results of the actual need for several experiments. 


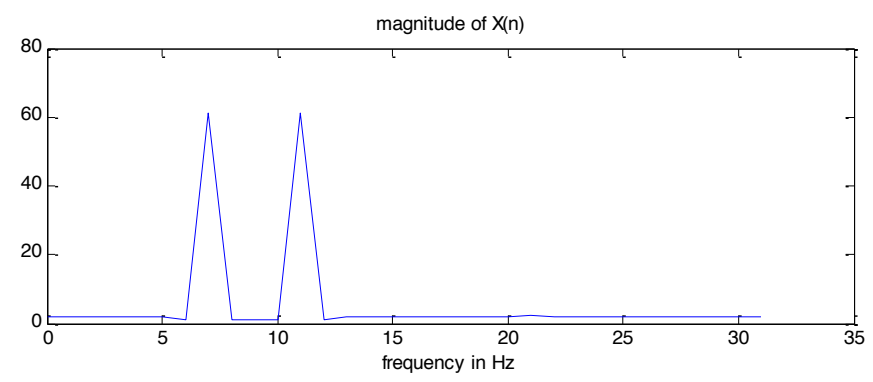

(a) FFT spectrum analysis

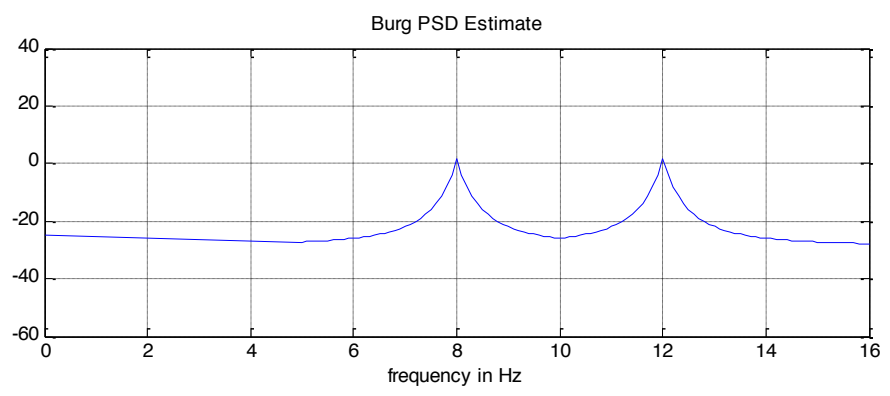

(b) $P_{1}=P_{2}=6$

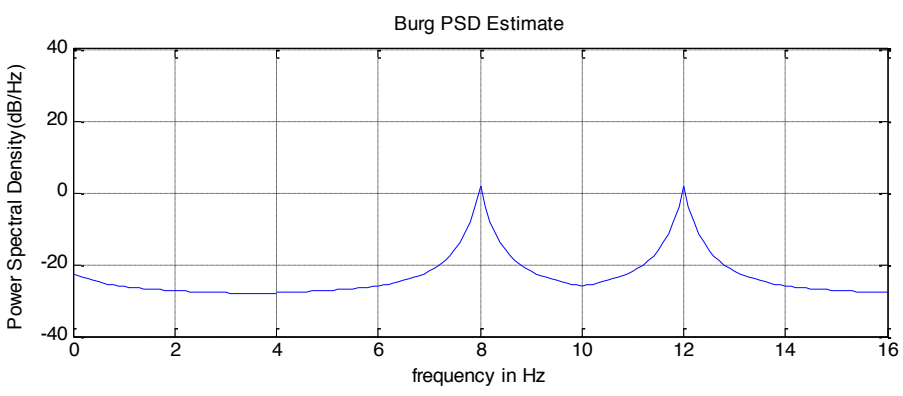

(c) $P_{3}^{\prime}=5$

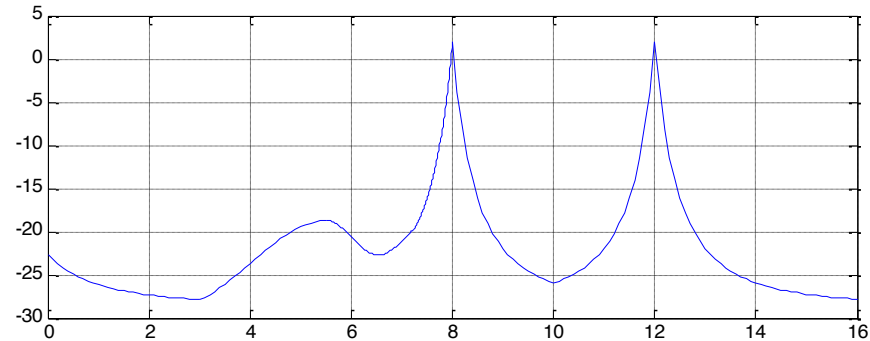

(d) $P_{3}^{\prime}=10$

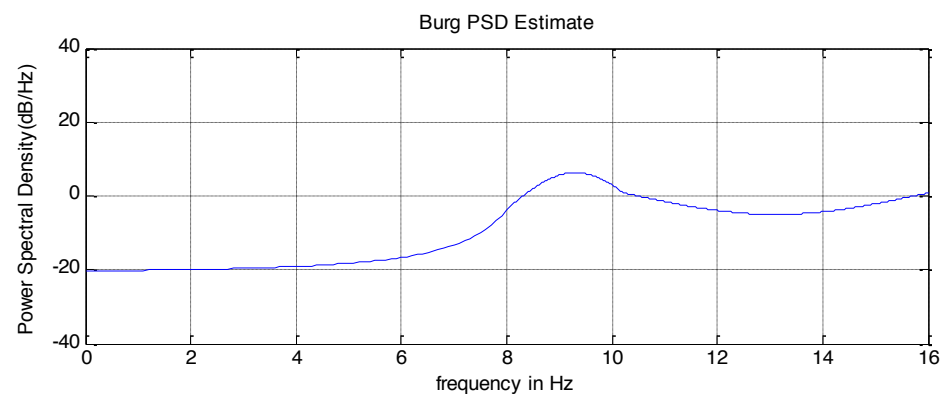

(e) $P_{4}^{\prime}=3$

Fig. (5). Power spectral analysis method under different criteria Burg. 


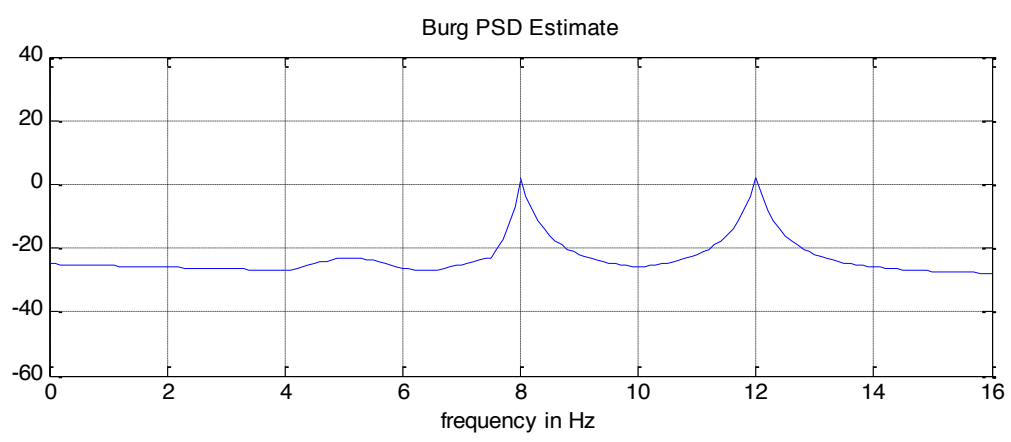

(a) $r=11$

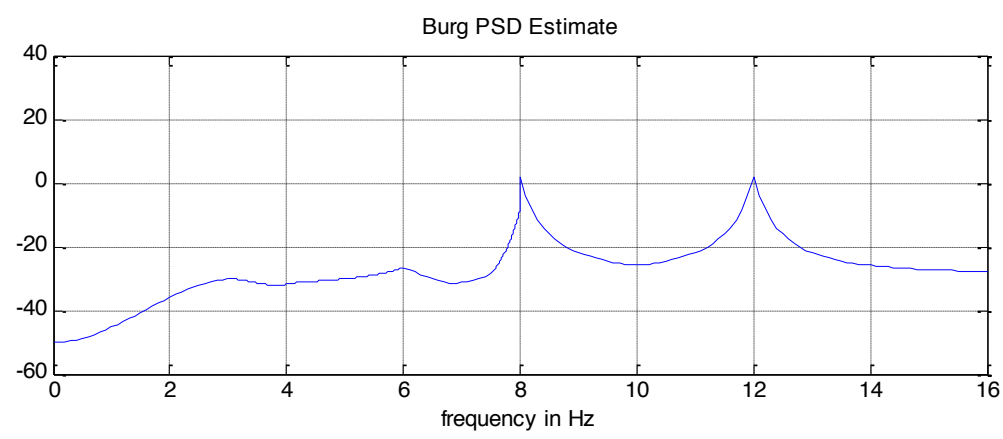

(b) $r=12$

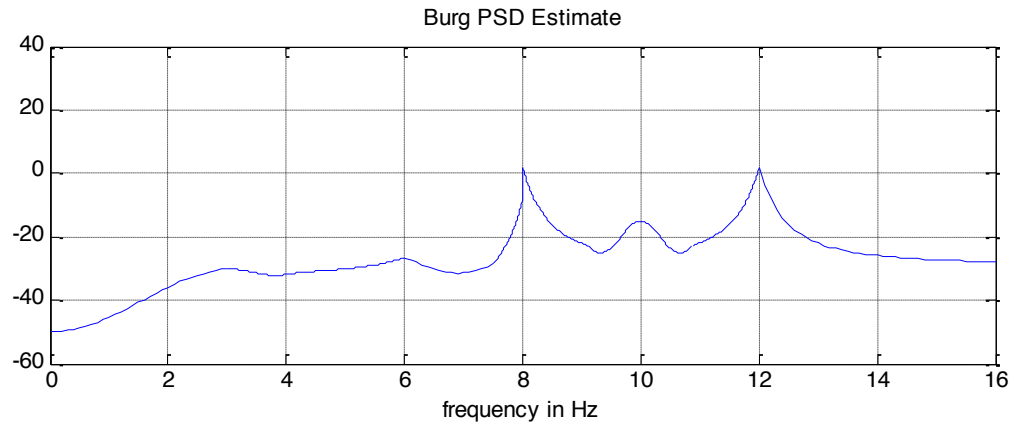

(c) $r=13$

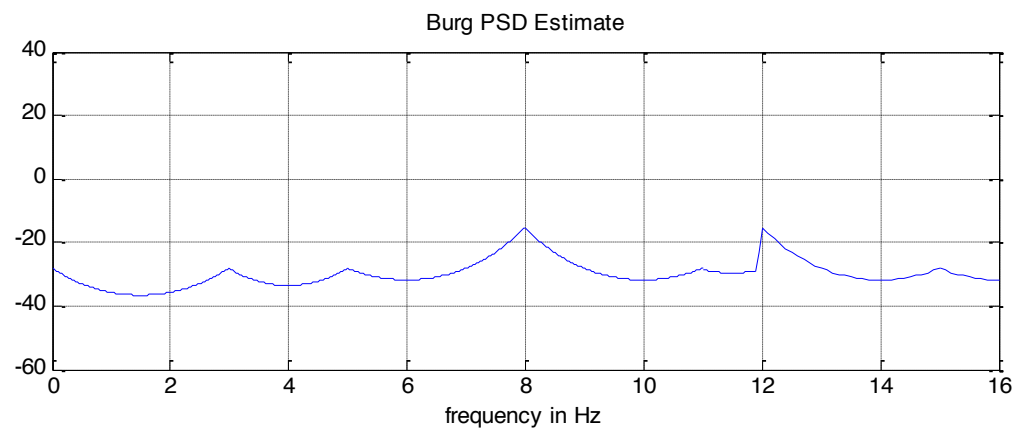

(d) $r=14$ 


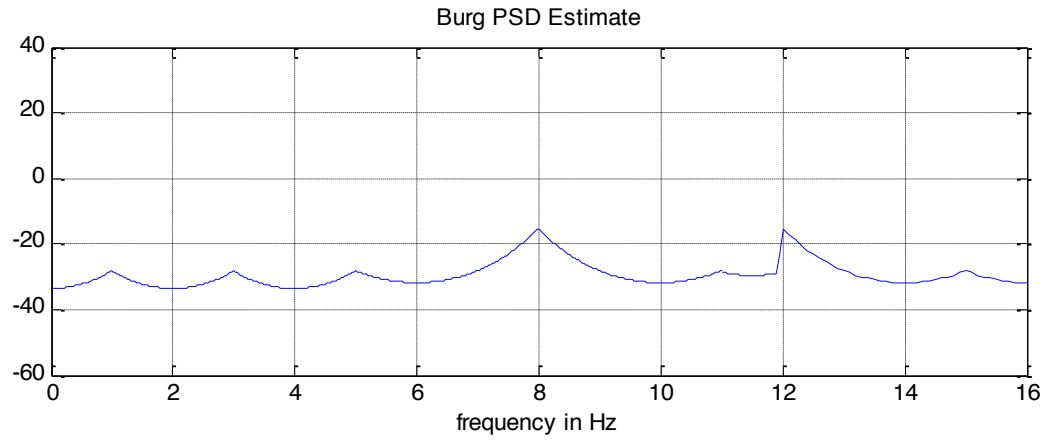

(e) $r=15$

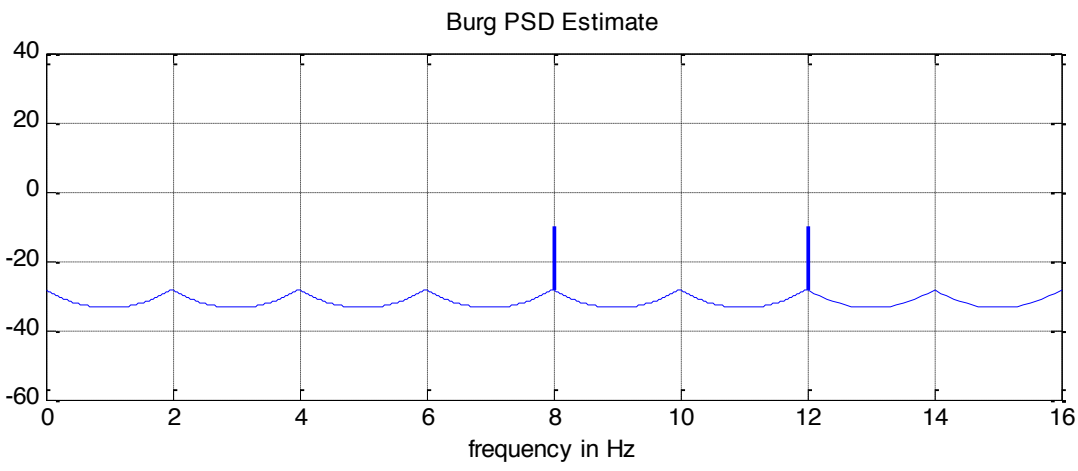

(f) $r=16$

Fig. (6). Different orders of power spectrum estimation.

\section{CONFLICT OF INTEREST}

The authors confirm that this article content has no conflicts of interest.

\section{ACKNOWLEDGMENTS}

This work is supported by the universities of Hebei province science and technology research projects No. Z2014011.

\section{REFERENCES}

[1] M.N. Huhns, and M.P. Singh, "Research Directions for ServiceOriented Multiagent Systems", IEEE Internet Computing, IEEE Computer Society, 2005.

[2] Y. Liu, A.H.H. Ngu, and L. Zeng, "QoS Computation and Policing in Dynamic Web Service Selection", IEEE Computer Scociety, ACM Press, New York, 2004.

[3] E.F. Wang, Z. Wang, J. Ma, and Q. Ding, "A New Method of Time-frequency Synthesis of Harmonic Signal Extraction from Chaotic Background", In: Special Issue on Selected Best Papers of the International Workshop CSEEE 2011, vol. 6, no. 7, 2011.

[4] Q. Huang, S. Su, S. Xu, J. Li, P. Xu, and K. Shuang, "Migrationbased elastic consolidation scheduling in cloud data center", In: Proceedings of IEEE ICDCSW, 2013.

[5] S. Srirama, O. Batrashev, and E. Vainikko, "Scicloud: scientific computing on the cloud", In: Proceedings of the 2010 10th IEEE/ACM International Conference on Cluster, Cloud and Grid Computing, IEEE Computer Society, 2010, pp. 579-580.

[6] C. Feier, D. Roman, A. Polleres, J. Domingue, M. Stollberg, and D. Fensel, "Towards intelligent web services: the web service modeling ontology (wsmo)", In: International Conference on Intelligent Computing (ICIC), 2005.

[7] Q. Huang, K. Shuang, P. Xu, J. Li, X. Liu, and S. Su, "Predictionbased Dynamic Resource Scheduling for Virtualized Cloud Systems", Journal of Networks, vol. 9, no. 2, pp. 375-383, 2014.
[8] D.R. Farrier, "Introduction to High Resolution Array Spectrum Estimation", Numerical Linear Algebra, Digital Signal Processing and Parallel Algorithms NATO ASI Series, vol. 70, pp. 495-500, 1991.

[9] X. Zhang, G. Pietsch, and E.M. Gockenbach, "Investigation of the thermal transfer coefficient by the energy balance of fault arcs in electrical installations", IEEE Transactions on Power Delivery, vol. 21, no. 1, pp. 425-431, 2006.

[10] N. Yunlu, and C. Hang, "Detection of Underwater Carrier-Free Pulse based on Time-Frequency Analysis", Journal of Networks, vol. 8, no 1, pp. 205-212, 2013.

[11] Z.H. Jing, J. Hua, and X.N. Yang, "A new method for detecting negative SNR UWB Signal", Communication Countermeasures, vol. 4, pp.17-21, 2007.

[12] P. Kathirvel, M.S. Manikandan, P. Maya, and K.P. Soman, "Detection of power quality disturbances with overcomplete dictionary matrix and 1-norm minimization,", In: 2011 International Conference on Power and Energy Systems(ICPS), pp. 1-6, 2011.

[13] $\mathrm{X} . \mathrm{Li}$, and $\mathrm{H}$. Yao, "Improved signal processing algorithm based on wavelet transform", Journal of Multimedia, vol. 8, no. 3, pp. 226232, 2013.

[14] J.G. Ma, M.X. Xi, Y.M. Lin, and Y.P. Li, "Chinese Internet routerlevel hop count measurement and analysis," Application Research on Computer, vol. 25, no. 27, pp. 2112-2114, 2008.

[15] H. Y. Kong, and H. V. Khuong, "Performance analysis of spacetime block coded cooperative wireless transmission in Rayleigh fading channels," KICS JCN, vol. 8, pp. 306-312, 2006.

[16] K. Xiong, Z.D. Qiu, H.K. Zhang, C.S. Li, and H.C. Chao,“A scalable fast forwarding approach for IP networks", International Journal of Internet Protocol Technology, vol. 3, no. 2, pp.119-127, 2008.

[17] H. M. Park, Y.I. Min, J.S. Han, and H.J. Choi, "Enhanced detection method for 3GPP LTE QO-SFBC system in frequency selective Rayleigh fading channel environment", ICUIMC, pp. 558-562, 2010.

[18] X. Li and H. Yao, "Improved signal processing algorithm based on wavelet transform", Journal of Multimedia, vol. 8, no. 3, pp. 226$232,2013$. 
[19] C.V. Hollot, V. Misra, D. Towsley, and W. Gong, "Analysis and design of controllers for AQM routers supporting TCP flows", IEEE Transactions on Automatic Control, vol. 47, no. 6, pp. 945959, 2002.

[20] Y. Min, H. Park, J. Jang, K. Kim, and H. Choi, "The enhanced decoding method for qo-sfbc system in frequency selective fading channel environment", Journal of Networks, vol. 6, no. 3, pp. 378386, 2011.

[21] H.K. Hoidalen, and M. Runde, "Continuous monitoring of circuit breakers using vibration analysis", IEEE Transactions on Power Delivery, vol. 20, no. 4, pp. 2458-2465, 2005.
[22] M. Landry, F. Lonard, and R. Beauchemin, "An improved vibration analysis algorithm as a diagnostic tool for detecting mechanical anomalies on power circuit breakers", IEEE Transactions on Power Delivery, vol. 23, no. 4, pp. 1986-1994, 2008.

[23] J. Huang, X.G. Hu, and X. Geng, "An intelligent fault diagnosis method of high voltage circuit breaker base on improved EMD energy entropy and multi-class support vector machine", Electrical Power System Research, vol. 81, no. 2, pp. 400-407, 2011.

Received: September 22, 2014

(C) Fu Chao; Licensee Bentham Open.

This is an open access article licensed under the terms of the Creative Commons Attribution Non-Commercial License (http://creativecommons.org/licenses/by-nc/3.0/) which permits unrestricted, non-commercial use, distribution and reproduction in any medium, provided the work is properly cited. 